\title{
Dual-Polarized Synthetic Antenna Array for GNSS Handheld Applications
}

\author{
V. Dehghanian, ${ }^{1,2}$ A. Broumandan, ${ }^{1}$ M. Zaheri, ${ }^{1}$ and J. Nielsen ${ }^{1}$ \\ ${ }^{1}$ Position Location and Navigation (PLAN) Group, University of Calgary, Calgary, AB, Canada T2N 1N4 \\ ${ }^{2}$ Department of Math, Physics and Engineering, Mount Royal University, Calgary, AB, Canada T3E 6K6 \\ Correspondence should be addressed to V. Dehghanian; vdehghanian@mtroyal.ca
}

Received 26 September 2012; Accepted 18 December 2012

Academic Editors: A. Maaref and G. Mazzini

Copyright (c) 2013 V. Dehghanian et al. This is an open access article distributed under the Creative Commons Attribution License, which permits unrestricted use, distribution, and reproduction in any medium, provided the original work is properly cited.

\begin{abstract}
Small portable Global Navigation Satellite System (GNSS) receivers have revolutionized personal navigation through providing real-time location information for mobile users. Nonetheless, signal fading due to multipath remains a formidable limitation and compromises the performance of GNSS receivers. Antenna diversity techniques, including spatial and polarization diversity, can be used to mitigate multipath fading; however, the relatively large size of the spatially distributed antenna system required is incompatible with the small physical size constraints of a GNSS handheld receiver. User mobility inevitably results in motion of the handset that can be exploited to achieve diversity gain through forming a spatially distributed synthetic array. Traditionally, such motion has been construed as detrimental as it decorrelates the received signal undermining the coherent integration processing gain generally necessary for acquiring weak faded GNSS signals. In this paper the processing gain enhancement resulting from a dual-polarized synthetic array antenna, compatible with size constraints of a small handset that takes advantage of any user imposed motion, is explored. Theoretical analysis and experimental verifications attest the effectiveness of the proposed dual-polarized synthetic array technique by demonstrating an improvement in the processing gain of the GNSS signal acquisition operation.
\end{abstract}

\section{Introduction}

The initial GNSS signal acquisition is a necessary step in the process of obtaining a set of pseudorange estimates sufficient for location estimation. The underlying multihypothesis detection problem is typically exasperated by a large search space which limits the processing resources and time that can be appropriated for testing individual hypotheses [1, 2]. Multipath fading, in the form of spatial signal power fluctuations [3], poses a formidable challenge to GNSS signal acquisition, a problem which has recently attracted significant interest $[4,5]$. Utilizing a higher processing gain through a longer integration time is not an attractive solution as the mean acquisition time is significantly increased [6]. In addition, signal decorrelation due to oscillator instability and user mobility effectively limits the coherent integration time [7].

Recently multiple receive antennas have been utilized to enhance signal detection performance in the form of beamforming and diversity systems $[8,9]$. Antenna diversity based on employing multiple antennas with different radiation characteristics has long been in use in diversity systems $[10,11]$. It can be shown that for typical indoor multipath environments resembling a sphere of scatterers model, an antenna separation as small as half a carrier wavelength results in a negligible signal complex correlation coefficient $[12,13]$. Even with this small element spacing, the size of a multielement antenna array is not compatible with the small physical size constraints of the GNSS handheld terminal.

Diversity achievable through approximately collocated antennas of differing polarization responses is an attractive option for the size constrained handset receiver [11, 14, 15]. Most of the work on polarization diversity is based on orthogonally polarized antennas $[11,16]$. In a polarization diversity system, colocated antennas with orthogonal polarizations are considered as uncorrelated diversity branches, provided that mutual coupling is minimal $[11,14,16]$. A twobranch antenna diversity system based on combining outputs of a Right-Hand Circularly Polarized (RHCP) antenna and a Left-Hand Circularly Polarized (LHCP) antenna can be 
considered for GNSS applications [17]. Although polarization diversity based on the circularly polarized GNSS signal provides two orthogonal polarizations, a higher number of diversity branches are typically desirable for further enhancements of the processing gain $[5,18]$. Spatial diversity can provide these additional diversity branches.

As stated earlier, utilizing spatial diversity through an array of spatially distributed antennas is not compatible with the size factor of GNSS handheld terminals. However, an innate property of the mobile receiver is that it is generally undergoing some form of motion as part of the typical usage mode. While such motion is typically regarded as being detrimental as it results in signal decorrelation limiting the processing gain achievable by coherent signal integration, it can be exploited to achieve usable diversity gain. The resulting diversity gain can enhance the performance of GNSS signal acquisition in multipath faded environments. Authors in $[5,18]$ have proposed a technique that utilizes spatial diversity based on motions of a single antenna and forming a spatially distributed synthetic array. Although the concept of synthetic array for signal parameter estimation has been around for several years (see [19]), its application for combating multipath fading was first introduced by [18].

Although a unipolarized synthetic array (the previous work, [5], which is herein called UPSA) enhances GNSS signal acquisition, capturing the required number of signal samples in spatially correlated channels requires longer trajectories of several carrier wavelengths. Nondeliberate motion on the part of the user may not naturally provide a trajectory of such length. To utilize shorter trajectories it is necessary to incorporate another form of diversity system in addition to the spatial diversity available. This paper proposes a combination technique based on merging polarization and spatial diversities through utilizing a dual-polarized RHCPLHCP antenna in a GNSS handheld receiver and exploiting user movements to form a dual-polarized synthetic array, herein called DPSA (see Figure 1).

Note that the use of a dual-polarized antenna in a handheld has previously been reported in the literature (see [11]); nevertheless, the detection performance of a moving dual-polarized antenna in the context of GNSS signal acquisition has not been quantified, before. This paper studies the effect of motion on the detection performance of a GNSS receiver based on a dual-polarized antenna and proposes a practical scheme for producing useful diversity gain. Here, the detection performance of the DPSA technique is compared to the previously proposed configuration, UPSA, and it is shown that a DPSA configuration devours a much shorter trajectory compared to UPSA, for given performance requirements. Also, through extensive theoretical analysis and experiments, it is shown that under similar conditions (trajectory length, wireless channel condition, etc.), DPSA outperforms UPSA and significantly improves the initial acquisition performance. The remainder of the paper is organized as follows.

Section 2 defines the system model and the assumptions. The dual-polarized synthetic array model and the despreading process are also presented in this section. Section 3 discusses the realizable detection enhancement through

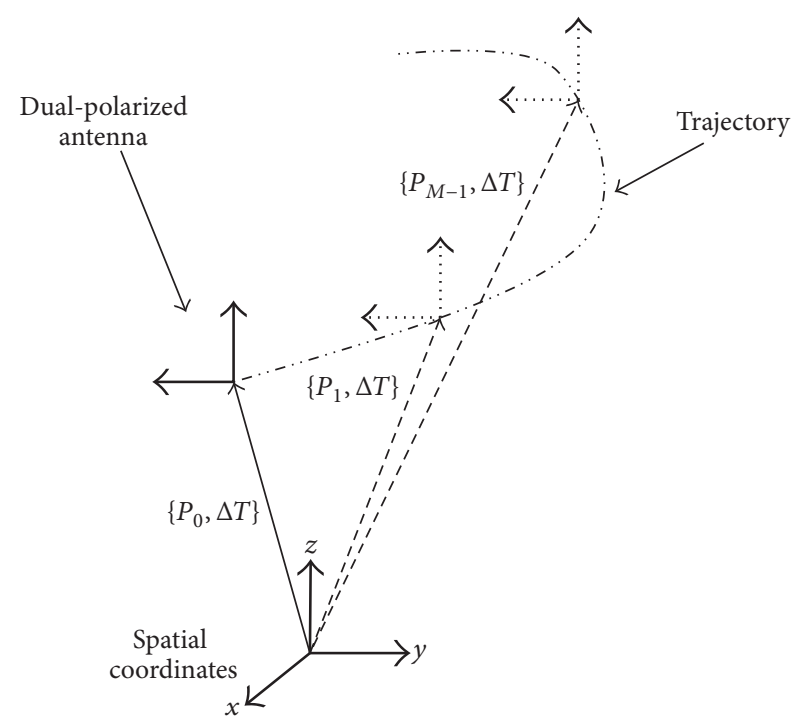

FIGURE 1: Dual-polarized synthetic array configuration.

comparing the performance of the proposed DPSA with that of the UPSA in a generalized correlated Rayleigh fading channel. Section 4 discusses the measurement results. Conclusions are given in Section 5.

\section{Assumptions and System Model}

The received complex GNSS baseband signal is denoted here by $r(t)$. The signal component of $r(t)$ emanating from the $k$ th satellite is represented by $s^{(k)}(t, \mathbf{p}(t))$ which is a function of time, $t$, as well as antenna position $\mathbf{p}(t)$. $\mathbf{p}(t)$ denotes the position vector of the phase center of the receiver antenna. $s^{(k)}(t, \mathbf{p}(t))$ can be decomposed to $s^{(k)}(t, \mathbf{p}(t))=$ $A^{(k)}(\mathbf{p}(t)) s_{o}^{(k)}(t)$ where $s_{o}^{(k)}(t)$ is the complex baseband component of the $k$ th satellite signal and is known to the receiver. $A^{(k)}(\mathbf{p}(t))$ is the channel response to the incident signal at the antenna position of $\mathbf{p}(t)$. The received signal, $r(t)$, is corrupted with additive noise which has an equivalent complex baseband representation denoted by $w(t)$. It is assumed that $w(t)$ is a complex normal random process, independent of the signal, and has a Power Spectral Density (PSD) that is constant within the bandwidth of the received signal. Herein, the signal detection of individual GNSS signals will be considered, while ignoring the presence of other GNSS signals. This is reasonable since the GNSS signal level is well below the noise floor, thus cumulative interference from other GNSS satellites is negligible. Hence, in the remainder of this paper, for the notational convenience, the superscript " $k$ " associated with individual satellites is omitted and the received signal is given by

$$
r(t)=A(\mathbf{p}(t)) s_{o}(t)+w(t),
$$

where

$$
s_{o}(t)=d(t-\tau) c(t-\tau) e^{j(2 \pi \Delta f t+\psi)} .
$$


$d(t)$ is the navigation data modulation, $c(t)$ is the Pseudorandom Noise (PRN) code, $\tau$ is the code phase, $\Delta f$ represents carrier frequency offset (due to the Doppler of the GNSS signal as well as any frequency offset of the receivers local oscillator), and $\psi$ is the initial phase offset. $s_{o}(t)$ is known to the receiver except for the navigation data, the code phase, the carrier frequency offset, and the initial phase offset $\psi$. For indoor GNSS applications it can be shown that $s_{o}(t)$ is sufficiently narrowband such that a flat fading model can be assumed $[3,5]$. It is also assumed that during the data collection, hereafter denoted by the signal snapshot period, $T$, the channel is temporally stationary, and therefore coherent integration results in positive processing gain. Consequently, during the snapshot interval, $T$, the channel gain, $A$, is assumed to be a function of position only, not time.

2.1. Synthetic Array Signal Model. In this subsection the utilized synthetic array processing is discussed. A stopmeasure-and-go data collection scenario is assumed, that is, the antenna remains static in each signal integration subinterval which here is denoted by $\Delta T=T / M$ where $M$ is the number of signal samples. Suppose that a GNSS receiver integrates a temporal snapshot of $r(t)$ over the interval of $t \in[0, T]$. Assume that each discrete sample is derived by despreading $r(t)$ with a locally generated signal for a subinterval duration of $\Delta T$. Define the $m$ th subinterval that extends over the time interval of $t \in\left[t_{m}, t_{m}+\Delta T\right]$ where $t_{m}$ is the starting instance of the $m$ th subinterval. In the UPSA scenario, the signal captured in each subinterval is correlated with $s_{o}(t)$ resulting in a set of $M$ spatial array samples. The $m$ th synthetic array sample is denoted by $x_{m}$ and given by

$$
\begin{aligned}
x_{m}= & \frac{1}{\Delta T} \int_{t_{m}}^{t_{m}+\Delta T} r(t) s_{o}(t)^{*} d t \\
= & \frac{1}{\Delta T} \int_{t_{m}}^{t_{m}+\Delta T} A(\mathbf{p}(t)) s_{o}(t) s_{o}(t)^{*} d t \\
& +\frac{1}{\Delta T} \int_{t_{m}}^{t_{m}+\Delta T} w(t) s_{o}(t)^{*} d t .
\end{aligned}
$$

Consider a normalization of the signal power such that $\int_{t}^{t+T}\left|s_{o}(t)\right|^{2} d t=1$. It is assumed that the bandwidth of $s_{o}(t)$ is significantly larger than the inverse of the channel coherence time such that (3) can be approximated by

$$
\begin{aligned}
x_{m} & \approx \frac{1}{\Delta T} \int_{t_{m}}^{t_{m}+\Delta T} A(\mathbf{p}(t)) d t+\frac{1}{\Delta T} \int_{t_{m}}^{t_{m}+\Delta T} w(t) s_{o}(t)^{*} d t \\
& =s_{m}+w_{m},
\end{aligned}
$$

where $s_{m}$ and $w_{m}$ are the signal and the noise components of the $m$ th collected sample, $x_{m}$. To simplify the expressions to follow and without any loss of generality, the noise covariance is assumed to be normalized such that $w_{m} \sim$ $\mathrm{CN}(0,1)$, where $\mathrm{CN}\left(\mu, \sigma^{2}\right)$ is the circular normal distribution with $\mu, \sigma^{2}$ representing the complex mean and the variance, respectively. The variance of the signal component, $s_{m}$, after
$\Delta T$ seconds of coherent integration is $\sigma_{s}^{2} / M$. Note that $\sigma_{s}^{2}$ is the variance of the complex signal component after $T$ seconds of coherent integration. Consequently, a signal-to-noise ratio (SNR) can be defined as

$$
\eta=\sigma_{s}^{2} .
$$

Note that noise is assumed to be solely intrinsic and independent of the signal. Consequently, $x_{m}, s_{m}$, and $w_{m}$ can be represented as a vector by

$$
\begin{aligned}
\mathbf{x} & =\left[x_{1}^{p}, \ldots, x_{M}^{p}\right]^{\dagger}, \\
\mathbf{s} & =\left[s_{1}^{p}, \ldots, s_{M}^{p}\right]^{\dagger}, \\
\mathbf{w} & =\left[w_{1}^{p}, \ldots, w_{M}^{p}\right]^{\dagger},
\end{aligned}
$$

where " $\dagger$ " denotes a matrix transpose, the superscripts " $p$ " stands for the principle polarization, and $x_{m}^{p}$ denotes the $m$ th signal sample collected based on an RHCP antenna.

Similarly, the baseband signal samples collected based on a DPSA configuration can be represented by

$$
\begin{gathered}
\mathbf{x}=\left[x_{1}^{p}, \ldots, x_{M}^{p}, x_{1}^{o}, \ldots, x_{M}^{o}\right]^{\dagger}, \\
\mathbf{s}=\left[s_{1}^{p}, \ldots, s_{M}^{p}, s_{1}^{o}, \ldots, s_{M}^{o}\right]^{\dagger}, \\
\mathbf{w}=\left[w_{1}^{p}, \ldots, w_{M}^{p}, w_{1}^{o}, \ldots, w_{M}^{o}\right]^{\dagger},
\end{gathered}
$$

where " $o$ " stands for the orthogonal polarizations and $x_{m}^{o}$ denotes the $m$ th signal sample collected based on an LHCP antenna.

It is further assumed that the baseband output signals of the orthogonally polarized antennas are uncorrelated and identically distributed [20]. In other words $E\left[s_{m}^{p} s_{n}^{o *}\right]=0$ and $\operatorname{var}\left(s_{m}^{p}\right)=\operatorname{var}\left(s_{n}^{p}\right)$ for all $m, n=1, \ldots, M$. Here, $E[\cdot]$ represents an expectation operator and “*” is a complex conjugate.

2.2. Multipath Model. Multipath fading at the output of the receiver antenna in severe and isotropic multipath environments such as indoors can be modeled according to the "sphere of scatterers model" [20-24]. According to this model, the magnitude of the complex correlation coefficient, $\left|\rho_{c}\right|$, between complex baseband voltages at the output of two copolarized isotropic antennas with spatial separation of " $\Delta l$ " follows from $[23,24]$ as

$$
\left|\rho_{c}\right|=\left|\frac{\sin (2 \pi \Delta l / \lambda)}{(2 \pi \Delta l / \lambda)}\right|,
$$

where $\lambda$ is the carrier wavelength. Hence, according to (8), the received signal decorrelates by a half a carrier wavelength antenna displacement. Therefore, by moving an antenna along a " $D=M \lambda / 2$ " long smooth trajectory in a multipath fading environment, one can collect up to $M$ spatially uncorrelated signal samples $[5,18,20]$. Consider that these $M$ signal samples resemble $M$ diversity branches experiencing flat Rayleigh fading. (There is some loss in generality by 
ignoring the resolvable multipath and assuming flat fading. Nevertheless, the frequency selective fading results in an alternate source of diversity which can be considered in processing gain evaluations [25]).

\section{Detection Performance and Processing Gain Analysis}

This paper focuses on GNSS signal acquisition in a multipath faded environment. This generally implies a multihypothesis detection process as part of the general acquisition over a given search space. Generally as the hypotheses are tested with orthogonal despreading functions, the performance can be assessed by considering an individual hypothesis testing. In this paper $s_{o}(t)$ is defined to correspond to the despreading function for a specific individual hypothesis test such that the detection process is binary $[1,2]$. Therefore $\mathscr{H} 1$ represents the case when the incoming signal corresponds to the hypothesis associated with $\mathbf{x}=\mathbf{s}+\mathbf{w}$, and $\mathscr{H} 0$ represents the case when the incoming signal corresponds to the hypothesis associated with $\mathbf{x}=\mathbf{w}$. The approximation in the analysis is that it is assumed that for the $\mathscr{H} 1$ state, $s_{o}(t)$ corresponds exactly to the spreading signal of the incoming signal except for an arbitrary complex amplitude coefficient. Hence, under $\mathscr{H} 1$ it is assumed that the frequency and code phase of the incident signal is synchronized with $s_{o}(t)$. A more detailed analysis would include the incremental loss associated with a slight mismatch of the spreading signal of the incoming signal and $s_{o}(t)$. However, herein the objective is to compare the signal acquisition performances of the DPSA and UPSA techniques. Hence, this incremental additional loss which affects all cases in approximately the same way will not be considered.

The design of the detection algorithm is based on target values of the probability of detection $\left(P_{D}\right)$ and the probability of false alarm $\left(P_{F}\right)$ associated with the search hypothesis. Assuming that the statistics of the signal and noise components are known, for a given spatial extent of the synthetic array, the target detection performance requirements of $\left\{P_{F}, P_{D}\right\}$ map into a specific required average signal-to-noise ratio (SNR). Regardless of further algorithm details, for a given utilization detection scenario in terms of $P_{F}$ and $P_{D}$, the goal is to optimize the receiver processing such that the required average SNR is minimized. Consequently, the problem is modeled as one of choosing between $\mathscr{H} 0$, the noise-only hypothesis, and $\mathscr{H} 1$, the signal-present hypothesis as

$$
\begin{array}{cc}
\mathbf{x}=\mathbf{w} & \text { under } \mathscr{H} 0 \\
\mathbf{x}=\mathbf{s}+\mathbf{w} & \text { under } \mathscr{H} 1
\end{array}
$$

where, $\mathbf{x}, \mathbf{s}$, and $\mathbf{w}$ were defined earlier (see (5) and (7)). Under generalized correlated Rayleigh fading conditions, the distribution of $\mathbf{x}$ is according to a zero-mean jointly circular normal PDF with a covariance that is denoted by $\mathbf{C}_{\mathbf{x}}$. In other words, $\mathbf{x} \sim \mathrm{CN}\left(\mathbf{0}, \mathbf{C}_{\mathbf{x}}\right)$.

The signal vector $\mathbf{x}$, can be mapped into a single detection variable $z$, through a diversity combiner. Consequently, a decision is made based on comparing $z$ to a given threshold, $\gamma$, which is typically selected based on a constraint of $P_{F}[26]$.
As the carrier phase of the received signal is unknown, a square law combiner (SLC) is utilized to map $\mathbf{x}$ to $z$ as

$$
z=\mathbf{x}^{\mathrm{H}} \mathbf{x}=\sum_{i=1}^{M}\left|x_{i}^{p}\right|^{2}+\mu \sum_{i=1}^{M}\left|x_{i}^{o}\right|^{2},
$$

where $\mu=0$ corresponds to UPSA and $\mu=1$ to DPSA scenarios [21]. Accordingly, the measured $P_{F}$ and $P_{D}$ are related to the cumulative distribution function(CDF) of $z$ and to the threshold, $\gamma$, based on

$$
\begin{aligned}
& P_{F}(\gamma)=1-\left.\mathscr{F}(\gamma)\right|_{\mathscr{H} 0}, \\
& P_{D}(\gamma)=1-\left.\mathscr{F}(\gamma)\right|_{\mathscr{H} 1},
\end{aligned}
$$

where $\mathscr{F}$ denotes the CDF of $z$ under $\mathscr{H} 0$ or $\mathscr{H} 1$ [26]. Consequently, the corresponding values of average SNRs determined for a target $\left\{P_{F}, P_{D}\right\}$ will be compared in order to provide a measure of performance enhancement as

$$
G=10 \log _{10}\left(\frac{\eta_{\mathrm{UPSA} / \text { Static }}}{\eta_{\mathrm{DPSA}}}\right)(\mathrm{dB}),
$$

where $G$ is the realized gain and $\eta_{\text {DPSA }}, \eta_{\text {UPSA }}$, and $\eta_{\text {Static }}$ are the required SNRs for achieving $\left\{P_{F}, P_{D}\right\}$ based on a DPSA, UPSA, or static antenna scenarios, respectively.

\subsection{Performance Evaluation in Independent Rayleigh Fading} Channel. In this subsection, the performances of the UPSA, the DPSA, and those of a static antenna will be compared. Observing the statistics of SLC under uncorrelated branch variables is insightful due to the simplicity of resulting mathematical expressions. Specifically, the advantage of the DPSA model over the UPSA model can be readily realized by noting the achievable extra degrees of freedom at the SLC output chi-squared PDF which will be discussed shortly.

Assume a linearly polarized antenna is translated along a smooth $M \lambda / 2$-long trajectory during the signal snapshot interval of duration $T$ such that $M$ spatially uncorrelated samples can be collected (UPSA scenario). Consequently, it can be shown that $\mathbf{x} \mid \mathscr{H} 0 \sim \mathrm{CN}\left(\mathbf{0}, \mathbf{I}_{M}\right)$ under the noise-only hypothesis and $\mathbf{x} \mid \mathscr{H} 1 \sim \mathrm{CN}\left(\mathbf{0},\left(\eta_{\text {UPSA }} / M\right) \mathbf{I}_{M}+\mathbf{I}_{M}\right)$ under the signal-present hypothesis. Here, $\mathbf{I}_{M}$ denotes an $M$ by $M$ identity matrix. These samples can be combined using the SLC combiner of (10). Consequently the statistics of the detection variable $z$ is

$$
z_{\mathrm{UPSA}} \sim \begin{cases}\mathscr{H} 0: & \chi_{2 M}^{2}(0,0.5) \\ \mathscr{H} 1: & \chi_{2 M}^{2}\left(0, \frac{\eta_{\mathrm{UPSA}}}{2 M}+0.5\right)\end{cases}
$$

where $\chi_{N}^{2}\left(s^{2}, \sigma^{2}\right)$ denotes a chi-squared distribution with $N$ degrees of freedom ( $N$ DOF) with noncentrality parameter $s^{2}$ and the common variance of the corresponding Gaussian components $\sigma^{2}$.

In a DPSA scenario (Figure 1), a doublet of orthogonally polarized antennas is translated over the same trajectory such that 2 orthogonal sets of $M$ spatially uncorrelated samples can be measured. Consequently, it can be shown 
that $\mathbf{x} \mid \mathscr{H} 0 \sim \mathrm{CN}\left(\mathbf{0}, \mathbf{I}_{2 M}\right)$ under the noise-only hypothesis and $\mathbf{x} \mid \mathscr{H} 1 \sim \mathrm{CN}\left(\mathbf{0},\left(\eta_{\text {DPSA }} / M\right) \mathbf{I}_{2 M}+\mathbf{I}_{2 M}\right)$ under the signalpresent hypothesis. Therefore, the SLC output statistics, $z$, for DPSA is

$$
z_{\mathrm{DPSA}} \sim \begin{cases}\mathscr{H} 0: & \chi_{4 M}^{2}(0,0.5) \\ \mathscr{H} 1: & \chi_{4 M}^{2}\left(0, \frac{\eta_{\mathrm{DPSA}}}{2 M}+0.5\right) .\end{cases}
$$

The extra DOF realized for the DPSA setup is a simple evidence of performance improvement. Figure 2 demonstrates the realizable processing gain (12) of a DPSA in comparison with a static antenna for various values of $P_{D}$ and $M$. As shown in this figure, for any target detection requirement of $\left\{P_{F}, P_{D}\right\}$, there is a specific $M$ that results in a minimum required SNR and therefore a highest processing gain, $G$. Therefore, there is an optimum $M$ for which $G$ is maximum which here is denoted by $M_{\text {opt }}$. The existence of an optimum $M$ is due to the increase of diversity gain as well as coherence losses as $M$ increases [5]. As shown there is about $20 \mathrm{~dB}$ gain utilizing the DPSA algorithm for target detection $P_{D}=$ 0.99 and $P_{F}=0.01 . M_{\mathrm{opt}}$ for this scenario is seven, which maps into a reasonable trajectory size for the GNSS handheld applications with the trajectory of six half carrier wavelengths corresponding to a total trajectory length of $54-75 \mathrm{~cm}$ for typical GNSS frequencies.

Figure 3 demonstrates the optimum $M$ for different values of $P_{F}$ and $P_{D}$. As shown, the required number of spatial samples, $M_{\text {opt }}$, is lower for DPSA as compared with UPSA. In other words, DPSA devours a shorter trajectory than UPSA does for a given $\left\{P_{F}, P_{D}\right\}$. Figure 4 demonstrates the realizable processing gain of DPSA as compared with UPSA computed from (12). As can be seen from this figure, for lower $M$ and higher detection requirements, DPSA's performance is significantly higher than UPSA's. By increasing $M$ the performance of the DPSA converges to that of the UPSA. This is due to the fact that both techniques are taking advantage of diversity gain regardless of the source of the diversity (polarization or spatial diversity).

3.2. Performance Evaluation in Correlated Rayleigh Fading Channel. In this subsection, DPSA performance under correlated Rayleigh fading channel will be discussed and compared to those of the UPSA and the static antenna scenarios. As stated earlier, the received signal in a generalized correlated Rayleigh fading channel is distributed according to a zero-mean jointly circularly normal random variable. The covariance of $\mathbf{x}$ can be given by $\mathbf{C}_{\mathbf{x}}=\mathbf{C}_{\mathbf{s}}+\mathbf{I}_{2 M}$ under $\mathscr{H} 1$ and $\mathbf{C}_{\mathbf{x}}=\mathbf{I}_{2 M}$ under $\mathscr{H} 0$, where $\mathbf{C}_{\mathbf{s}}$ is the covariance of the signal component s. Consequently, it can be shown that the CDF of $z$ (see (10) for DPSA, $\mu=1$ ) scenario can be given by [26]

$$
\mathscr{F}_{\text {DPSA }}(\gamma)=\int_{-\infty}^{\gamma} d t \int_{-\infty}^{\infty} \prod_{i=1}^{M} \frac{1}{\left(1-j \lambda_{i} \omega\right)^{2}} \exp (-j \omega t) \frac{d \omega}{2 \pi},
$$

where $\lambda_{i}^{p}=\lambda_{i}^{o}=\lambda_{i}$ are the eigenvalues of the covariance matrix, $\mathbf{C}_{\mathbf{x}}$. Note that $\lambda_{i}^{p}=\lambda_{i}^{o}$ arises from the assumption of identical distribution of the signal in either polarizations. As

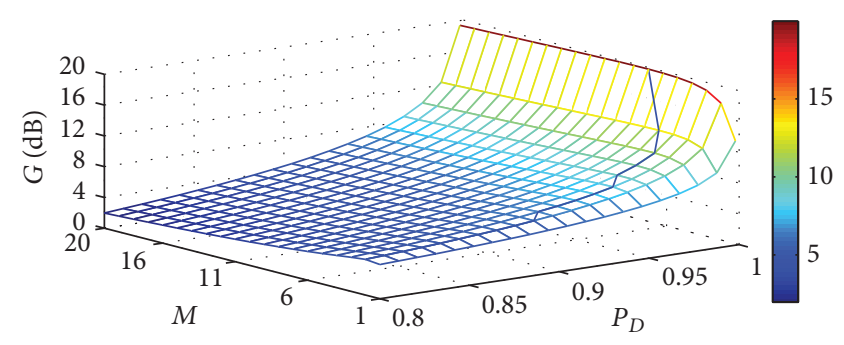

FIGURE 2: Processing gain of DPSA as compared with a static antenna for $P_{F}=0.01$.

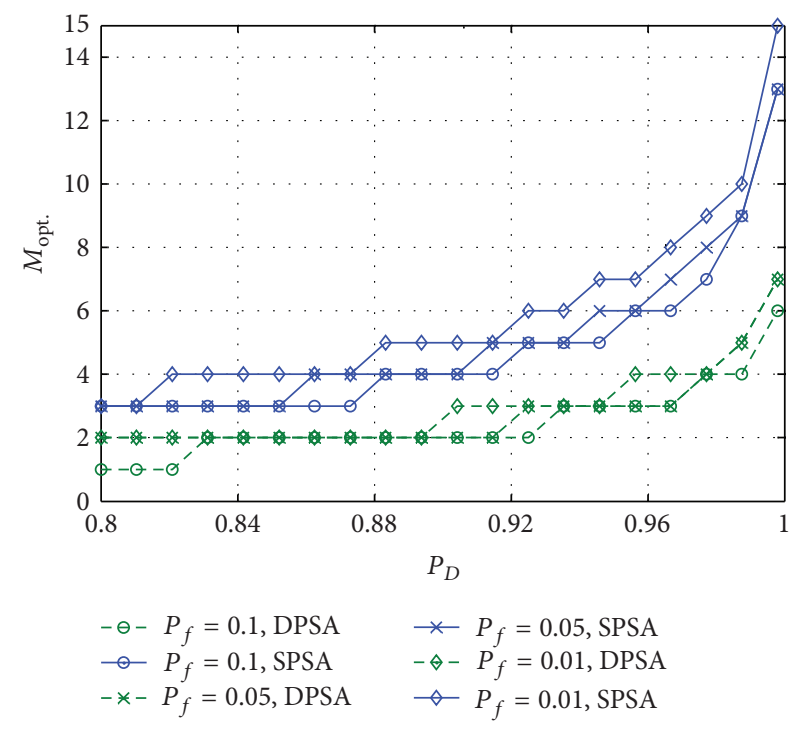

Figure 3: $M_{\mathrm{opt}}$ for different values of $P_{F}$ and $P_{D}$.

shown in the Appendix, if the nonzero eigenvalues of $\lambda_{i}$ are distinct, a closed form expression can be derived for the CDF of SLC output for DPSA scenario using the partial fraction expansion as [21]

$$
\mathscr{F}_{\mathrm{DPSA}}(\gamma)=1-\sum_{i=1}^{M}\left(A_{i}\left(1+\frac{\gamma}{\lambda_{i}}\right)+B_{i}\right) \exp \left(\frac{-\gamma}{\lambda_{i}}\right),
$$

where

$$
\begin{gathered}
A_{i}=\prod_{\substack{k=1 \\
k \neq i}}^{M} \frac{1}{\left(\lambda_{k} / \lambda_{i}-1\right)^{2}}, \\
B_{i}=A_{i} \sum_{\substack{m=1 \\
m \neq i}}^{M} \frac{-2 \lambda_{m}}{\lambda_{i}-\lambda_{m}} .
\end{gathered}
$$

In a UPSA configuration, the covariance of $\mathbf{x}$ can be given by $\mathbf{C}_{\mathbf{x}}=\mathbf{C}_{\mathbf{s}}+\mathbf{I}_{M}$ under $\mathscr{H} 1$ and $\mathbf{C}_{\mathbf{x}}=\mathbf{I}_{M}$ under $\mathscr{H} 0$, where $\mathbf{C}_{\mathbf{s}}$ is the covariance of the signal component $\mathbf{s}$. Consequently, it can be shown that the CDF of $z$ (see (10) for UPSA, $\mu=0$ ) is given by [26]

$$
\mathscr{F}_{\text {UPSA }}(\gamma)=\int_{-\infty}^{\gamma} d t \int_{-\infty}^{\infty} \prod_{i=1}^{M} \frac{1}{1-j \lambda_{i} \omega} \exp (-j \omega t) \frac{d \omega}{2 \pi},
$$




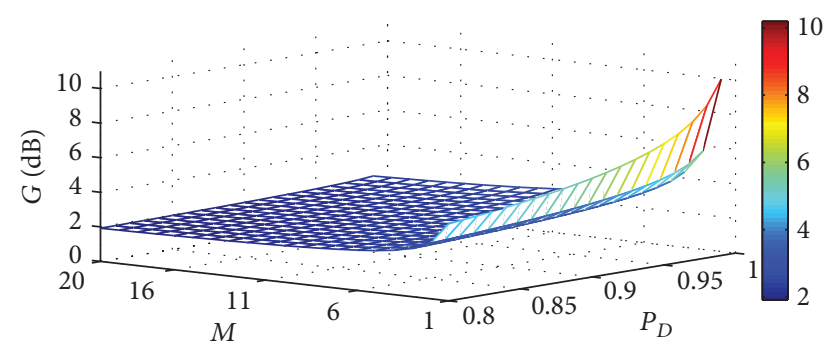

FIgURE 4: Processing gain $(G)$ of DPSA compared to UPSA for $P_{F}=$ 0.01 and different values of $M$ and $P_{D 0}$.

where $\lambda_{i}$ are the eigenvalues of $\mathbf{C}_{\mathbf{x}}$. Similar to the DPSA scenario, if the nonzero eigenvalues $\lambda_{i}$ are distinct, a closed form expression can be derived for the CDF of the SLC output as [26]

$$
\mathscr{F}_{\mathrm{UPSA}}(\gamma)=1-\sum_{i=1}^{M} T_{i} \exp \left(\frac{-\gamma}{\lambda_{i}}\right)
$$

where

$$
T_{i}=\prod_{\substack{k=1 \\ i \neq k}}^{M} \frac{1}{1-\lambda_{k} / \lambda_{i}} .
$$

As shown in (8) the correlation between two spatial diversity branches is a function of their spatial separation. Hence, the branch correlation can be controlled by selecting the synthetic array element spacing. Figure 5 demonstrates the achievable processing gain of DPSA compared to a static antenna for the specific target performance requirements of $P_{F}=0.01, P_{D}=0.99$ for different values of $M$ and antenna trajectory length, $D$. As shown in Figure 5 for a given $M$, increasing $D$ initially enhances the processing gain which is due to a decrease in branch correlation arising from longer array element spacing. However since a half-wavelength antenna spacing is enough to provide uncorrelated samples and to achieve full diversity gain, a longer trajectory, $D>$ $M \lambda / 2$, does not improve the detection performance.

In addition, it can be seen from Figure 5 that increasing $M$ for a specific $D$ can result in performance losses. This firstly arises from the fact that a larger $M$ necessitates a smaller integration subinterval, $\Delta T=T / M$, resulting in smaller processing gain. Secondly, increasing $M$ for a specific $D$ increases the correlation among spatial samples which reduces the potential diversity gain. As a result and similar to the uncorrelated scenario, for any $D$, there is an optimum $M$ for which the processing gain of DPSA is maximum. Figure 6 shows the realizable processing gain of the DPSA algorithm compared to UPSA for various values of $M$ and $D$. As shown in this figure, for smaller values of $M$ and $D$, DPSA significantly outperforms UPSA which is due to the significance of polarization diversity where the potential for spatial diversity is rather limited. Note that the performance of DPSA converges towards that of UPSA where the potential for spatial diversity is more available (i.e., larger $D$ and $M$ ).

Figure 7 is a plot of optimum $M$ as a function of trajectory length, $D$, for two different practical detection performance

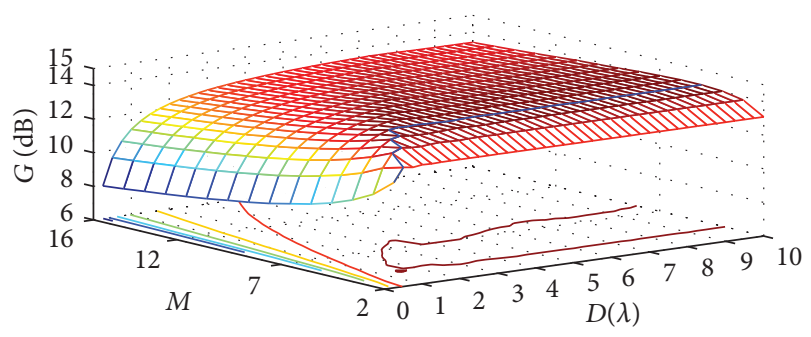

FIGURE 5: Processing gain of DPSA versus static antenna scenario for $P_{F}=0.01, P_{D}=0.99$.

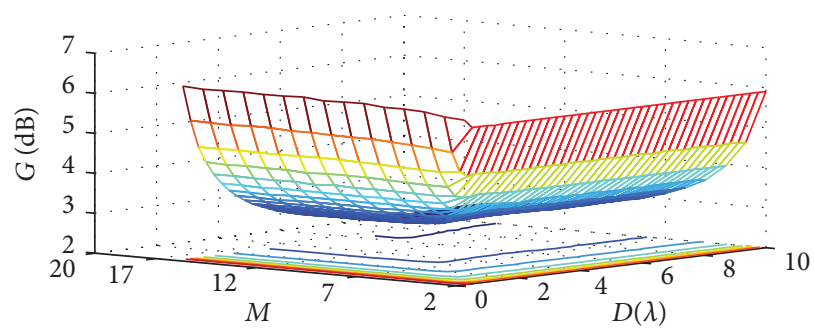

FIgURE 6: Processing gain of DPSA versus UPSA for $P_{F}=0.01$, $P_{D}=0.99$.

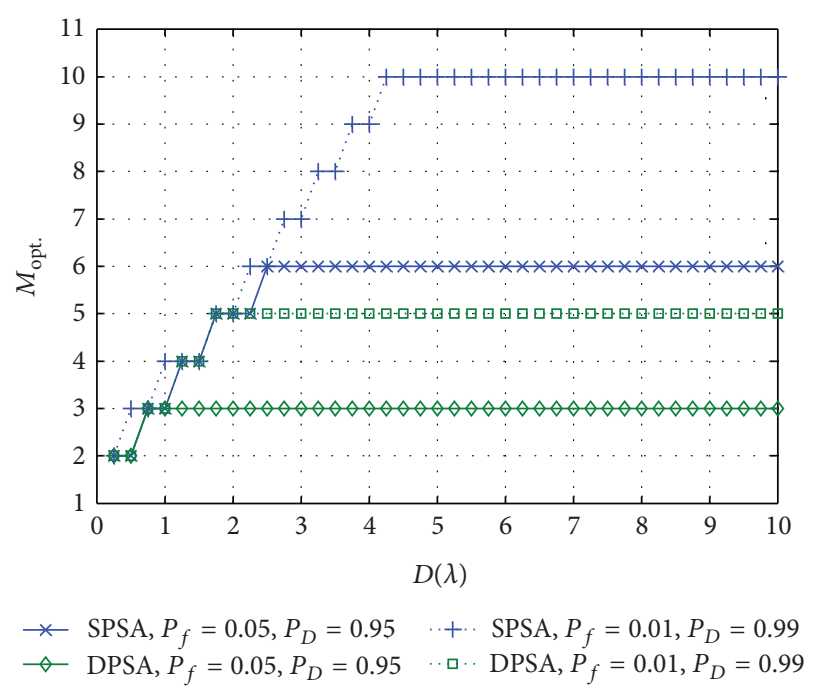

FIGURE 7: $M_{\text {opt }}$ versus trajectory length $(D)$.

requirements, $\left\{P_{F}=0.05, P_{D}=0.95\right\}$ and $\left\{P_{F}=0.01, P_{D}=\right.$ $0.99\}$. Similar to the spatially uncorrelated scenario of Figure 3 , a smaller $M_{\text {opt }}$ is realized by utilizing DPSA versus UPSA.

\section{Measurements and Analysis}

Experimental measurements represented in this section are based on capturing live GPS L1 C/A signals in an indoor environment. An extensive set of data was captured under controlled conditions, that is, constant antenna motion speed, in a large two and half story workshop as shown in Figure 8(a). Note that in practice a controlled motion on a known trajectory is not required and any form of user motion 


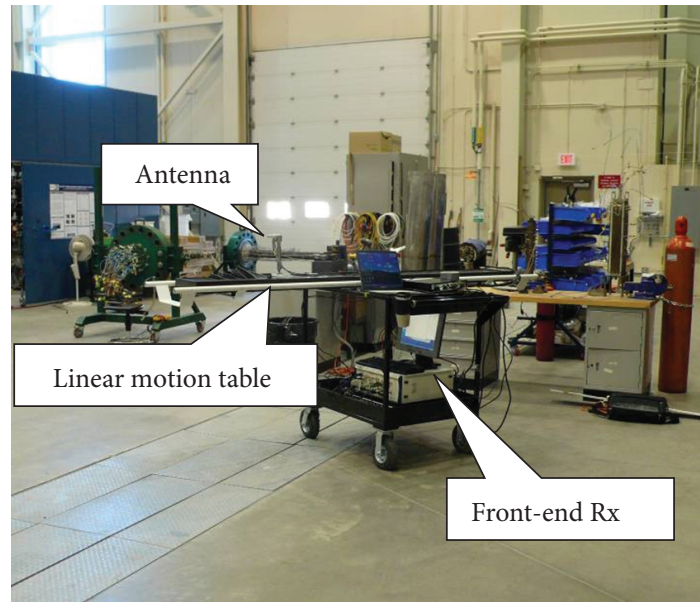

(a)

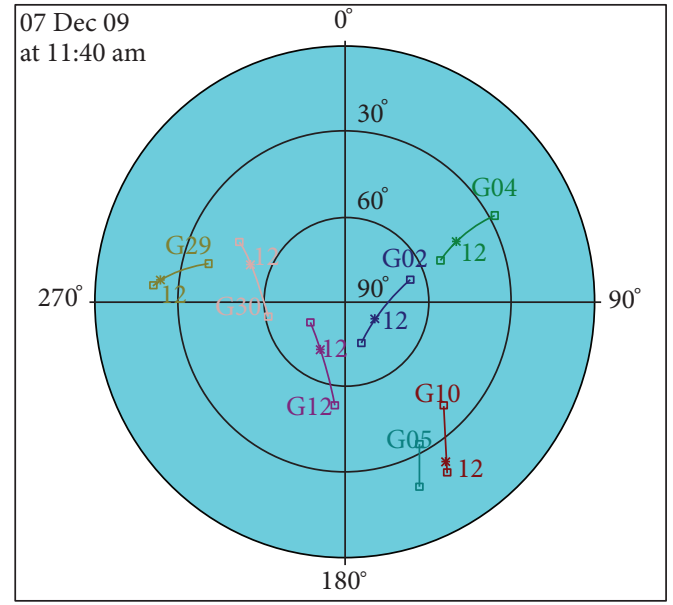

(b)

FIGURE 8: Data collection location and test setup and sky plot of available satellites.

can be utilized for achieving diversity. Figure 8(b) shows a sky plot of available satellites during the course of measurements. A layout of data collection site is also shown in Figure 9.

A dual polarized RHCP-LHCP active GPS antenna with a semispherical power pattern was mounted on a linear moving table to create the synthetic antenna array. The linear table was set to move the antenna with a constant speed of $2 \mathrm{~cm} / \mathrm{s}$. Since GPS signal is very weak inside buildings mainly due to the attenuation caused by walls and ceilings, a long coherent integration is required for initial acquisition. Therefore, the removal of navigation data is essential in order to facilitate this long coherent integration. As a result, a reference static antenna with a clear sky view of the GPS satellites was located outdoor to assist the navigation data removal of the indoor antennas. In other words, the estimated navigation data bits from the reference antenna were used to demodulate the indoor signals. The antennas were connected to a synchronous two-channel RF frontend which downconverts the GPS signal to baseband which is eventually sampled at a $10 \mathrm{MHz}$ rate after passing through commensurate antialiasing filters. The indoor receiver aiding process is shown in Figure 10.

Note that the complex baseband signal, $s_{i}$, is composed of fast and slow fading components as

$$
s_{i}=m_{i}\left(n_{i} e^{j \psi_{i}}\right) \quad \text { for } i=1, \ldots, M,
$$

where $j=\sqrt{-1}, \psi_{i}$ is the signal phase, $n_{i}$ is the signal envelope after local mean removal (the fast fading part of the signal), and $m_{i}$ represents the local-envelope mean (the slow-fading part of the signal [27]). $m_{i}$ is assumed to be constant over the course of measurements. In realistic cases, $m_{i} \neq m_{k}$ for $i \neq k$ and varies slowly due to path loss and shadowing [28]. Nonetheless, the received signal must be normalized to its envelope local mean in order to filter-out the slow-fading component of the signal $[11,14,27]$. This normalization is essential since it maps the received signal samples into samples of an ergodic process and consequently validates

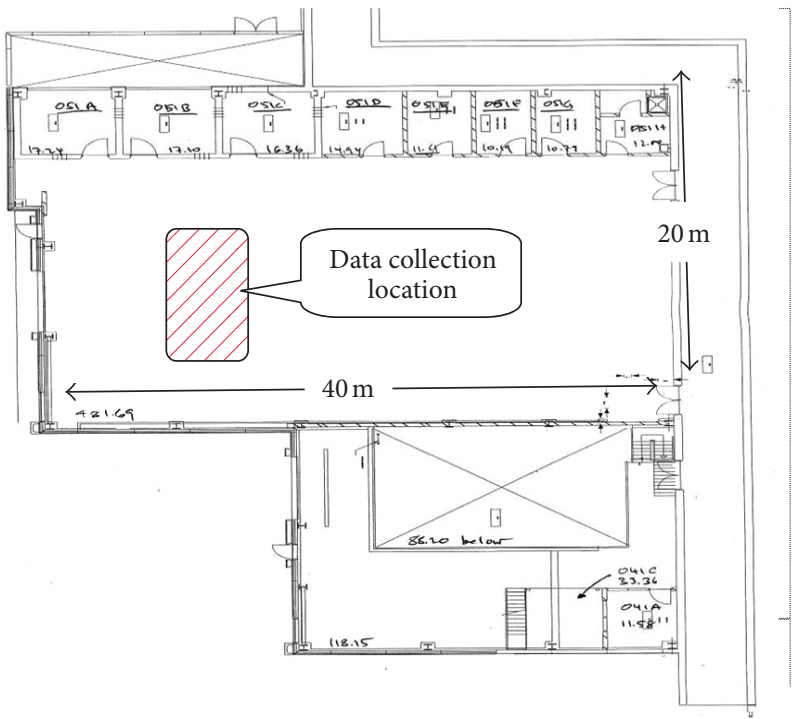

FIgURE 9: Floor plan of the measurement site.

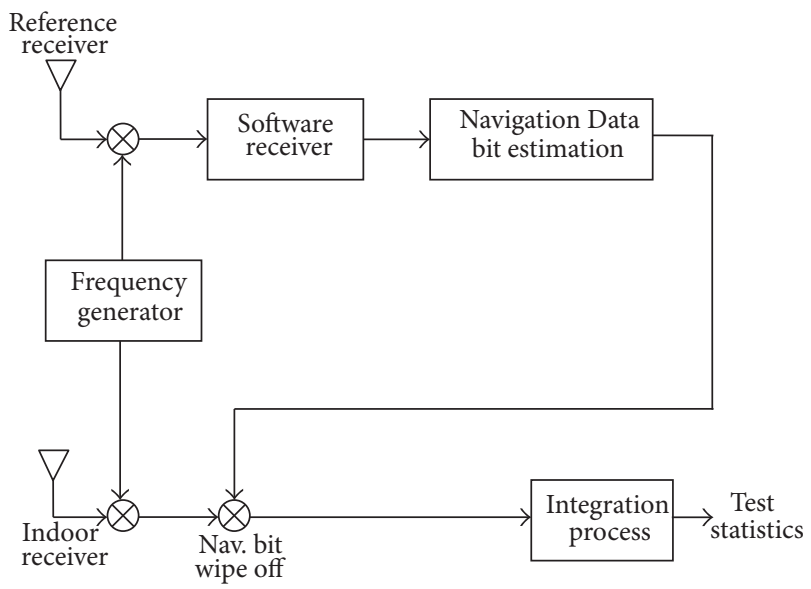

FIGURE 10: Navigation bit aiding process. 
the use of time average instead of expected value operator [21]. Hence, herein the received signal was normalized to its amplitude local mean in order to mitigate shadowing and path loss. In addition, a three-point moving average filter was applied to the processed signal to mitigate high frequency noise and digitizing errors [11]. The magnitude of complex correlation coefficient between the RHCP and LHCP branches was then calculated given the standard correlation coefficient estimation as

$$
\left|\rho_{c}\right|=\left|\frac{\left\langle\left[\mathbf{x}^{p}\right]\left[\mathbf{x}^{o}\right]^{H}\right\rangle}{\sqrt{\left\langle\left[\mathbf{x}^{p}\right]\left[\mathbf{x}^{p}\right]^{H}\right\rangle\left\langle\left[\mathbf{x}^{o}\right]\left[\mathbf{x}^{o}\right]^{H}\right\rangle}}\right|,
$$

where $\langle\cdot\rangle$ denotes a time average and " $H$ " is a matrix Hermitian. The results are demonstrated in Table 1. As can be seen from this table, there is negligible correlation between RHCP and LHCP signals. Note that the RHCP and LHCP signals are both coherently demodulated by the same clock such that the measured correlation is mainly a function of antenna polarizations.

Figure 11 shows the magnitude of complex correlation between spatially separated samples as a function of their spatial distance plotted for PRN 30. As can be seen from this figure, signal's spatial decorrelation is in good agreement with the theoretical model of (8) which is due to the nearly spherical distribution of scatterers in the measurement site.

Figure 12 shows a histogram of signal magnitude based on measurements under $\mathscr{H} 1$ state and its corresponding theoretical Rayleigh PDF for PRNs 4, 12, and 30. As can be seen from this figure, the measured histogram is in good agreement with the theoretical Rayleigh fading model assumed in this paper.

An SLC combiner was employed to combine a set of $M=3$ signal samples. Two different sample spacings of $\Delta l=\lambda / 2$ and $\Delta l=\lambda / 6$ were considered, and the signal snapshot period was selected to be $T=300 \mathrm{~ms}$. The Receiver Operating Characteristics (ROC) curves of Figure 13 provide a comparison between the performances of DPSA and UPSA and those of a static antenna. As can be seen from the ROC plots of Figure 13, a significant improvement in signal detection is realizable for DPSA as compared with UPSA and static antenna scenarios. As expected, DPSA always outperforms UPSA (the method which is used in [5]) due to the availability of an extra diversity branch at each spatial point. Also as can be seen from Figure 13, a smaller element spacing degrades the performances of both DPSA and UPSA due to a higher correlation between the available diversity branches.

Table 2 summarized the measured processing gain (DPSA versus UPSA) for $M=1,2,3$ based on a target performance requirement of $P_{F}=0.01, P_{D}=0.99$ for $D=$ $0.25,0.5,0.75(\lambda)$. Theoretical values of processing gains are also computed based on (12). Both RHCP and LHCP signals (denoted by $\mathrm{R}$ and $\mathrm{L}$ in the table) were considered for the UPSA scenario. It is evident from the measurement results that DPSA significantly outperforms UPSA. Note that $M=1$ corresponds to a static antenna scenario.
TABLE 1: Measured magnitude of complex correlation, $\left|\rho_{c}\right|$, between the received RHCP and LHCP signals after $100 \mathrm{~ms}$ of coherent integration.

\begin{tabular}{lccc}
\hline & PRN 30 & PRN 12 & PRN 04 \\
\hline$\left|\rho_{c}\right|$ & 0.08 & 0.18 & 0.21 \\
\hline
\end{tabular}

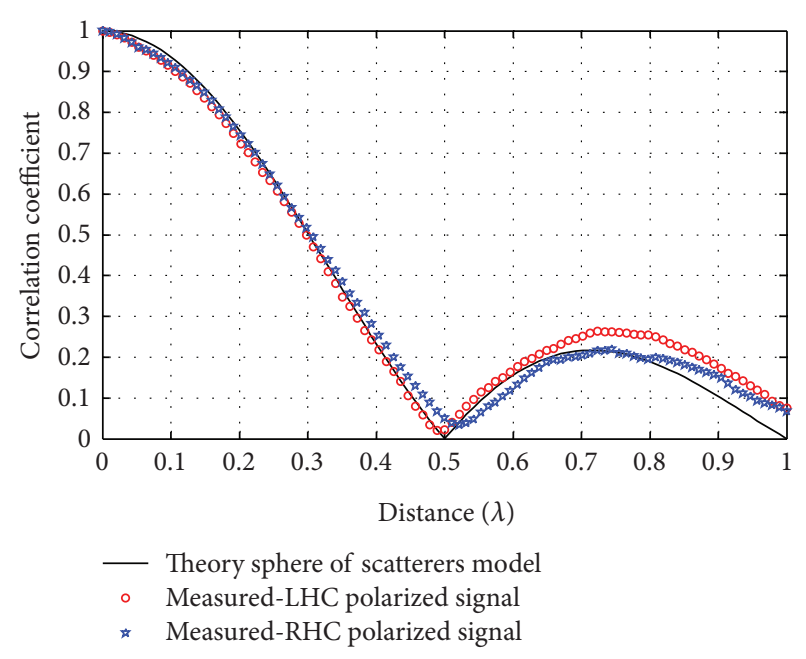

FIgURE 11: The magnitudes of complex spatial correlation of the received RHCP/LHCP signals (PRN 30).

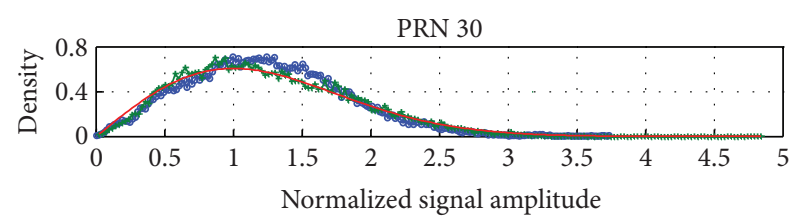

PRN 12
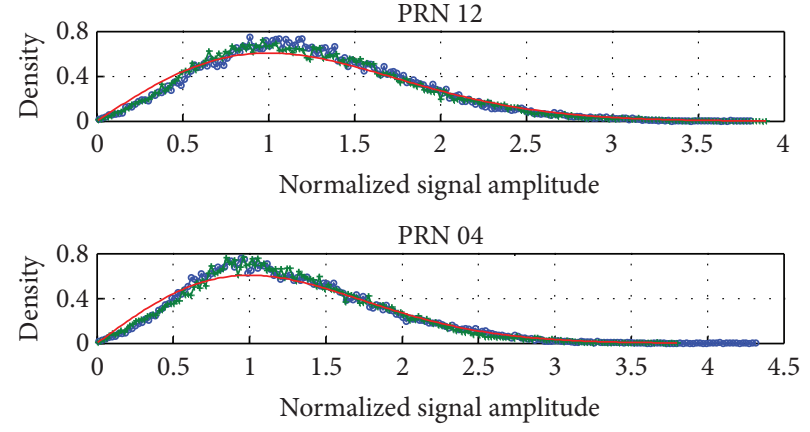

$\longrightarrow$ RHCP signal envelope
$\longrightarrow$ LHCP signal envelope

- Rayleigh PDF

FIGURE 12: Measured and theoretical Rayleigh PDF of signal magnitude.

\section{Conclusions}

In this paper the potential diversity gain resulting from the combination of using a small dual-polarized antenna that undergoes arbitrary spatial motion was considered for multipath mitigation in GNSS handheld receivers. Spatial movement of the antenna during signal collection, which is normally regarded as a detriment, was exploited to achieve 


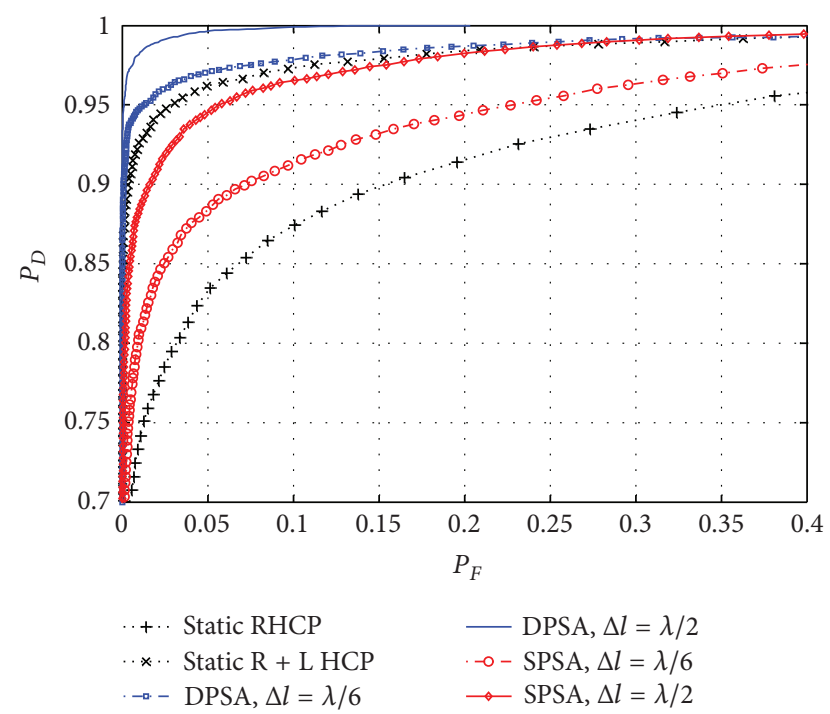

FIgURE 13: Measured ROC curves of DPSA, UPSA, and static antenna scenarios (PRN 30).

TABLE 2: Measured and theoretical processing gain of DPSA versus UPSA (R/L) for $P_{D}=0.99, P_{F}=0.01$.

\begin{tabular}{lccccccc}
\hline & \multicolumn{9}{c}{ Processing gain $(G)$} \\
$D(\lambda)$ & Theory & \multicolumn{2}{c}{ PRN 30 } & \multicolumn{2}{c}{ PRN 12 } & \multicolumn{2}{c}{ PRN 04 } \\
& & R & L & R & L & R & L \\
\hline \multicolumn{7}{c}{$M=1$} \\
N/A & 10.1 & 9.2 & 8.6 & 8.9 & 8.9 & 8.9 & 10.1 \\
N/A & 10.1 & 9.2 & 8.6 & 8.9 & 8.9 & 8.9 & 10.1 \\
N/A & 10.1 & 9.2 & 8.6 & 8.9 & 8.9 & 8.9 & 10.1 \\
\hline & & & $M=2$ & & & \\
0.25 & 6.0 & 4.8 & 4.1 & 4.8 & 5.1 & 5.0 & 6.4 \\
0.5 & 5.8 & 5.9 & 4.5 & 4.5 & 4.8 & 5.5 & 6.2 \\
0.75 & 6.0 & 5.7 & 4.6 & 5.0 & 3.9 & 4.9 & 5.5 \\
\hline & & \multicolumn{7}{c}{$M=3$} & & & \\
0.25 & 6.0 & 4.4 & 3.0 & 3.5 & 3.8 & 4.9 & 5.4 \\
0.5 & 5.2 & 4.3 & 3.7 & 3.4 & 3.2 & 3.8 & 5.0 \\
0.75 & 4.6 & 3.8 & 3.5 & 3.4 & 3.6 & 4.6 & 4.6 \\
\hline
\end{tabular}

additional processing gain through the added diversity. The arbitrary motion assumed was within the range of a typical usage case of the mobile device. The result was equivalent to a dual-polarized spatially distributed synthetic array that provides significant diversity gain even in a spatially correlated channel where the previously proposed UPSA technique is less effective. As shown, the required trajectory length for achieving a specific target detection requirement is much shorter for DPSA as compared to UPSA. This makes DPSA more compatible with realistic usage mode of a handset receiver. Theoretical analysis show that for a practical target detection requirement (e.g., $P_{D}=0.99, P_{F}=0.01$ ), a significant processing gain of about $10 \mathrm{~dB}$ can be achieved through utilizing DPSA, compared to UPSA. This processing gain is due to the extra degrees of freedom made available through polarization diversity in a DPSA configuration. The detection performance of DPSA was also compared to that of the UPSA under different channel conditions, and it was shown that the detection performance of DPSA significantly outperforms that of the UPSA especially when the channel is spatially correlated. Consequently, an extensive set of measurements utilizing indoor GPS signals were performed to verify the assumption of the fading channel and the estimated processing gains. The theoretical evaluations and the experimental analysis proved to be in good agreement. It is evident from these results that DPSA outperforms the UPSA by exploiting the additional polarization diversity available through the dual polarized antenna. This additional processing gain maps directly into an enhancement in GNSS signal acquisition.

\section{Appendix}

This appendix derives the CDF of summation of correlated central chi-squared random variables with four degrees of freedom based on the partial fraction expansion as

$$
\prod_{i=1}^{M} \frac{1}{\left(1-j \lambda_{i} \omega\right)^{2}}=\sum_{i=1}^{M} \frac{A_{i}}{\left(1-j \lambda_{i} \omega\right)^{2}}+\sum_{i=1}^{M} \frac{B_{i}}{\left(1-j \lambda_{i} \omega\right)},
$$

where

$$
\begin{gathered}
A_{i}=\left.\prod_{\substack{k=1 \\
k \neq i}}^{M} \frac{1}{\left(1-j \lambda_{k} \omega\right)^{2}}\right|_{\substack { \omega=-j / \lambda_{i} \\
\begin{subarray}{c}{k=1 \\
k \neq i{ \omega = - j / \lambda _ { i } \\
\begin{subarray} { c } { k = 1 \\
k \neq i } }\end{subarray}} ^{M} \frac{1}{\left(\lambda_{k} / \lambda_{i}-1\right)^{2}}, \\
\left.B_{i}=\left.\frac{d}{d \omega}\left(\prod_{\substack{k=1 \\
k \neq i}}^{M} \frac{1}{\left(1-j \lambda_{k} \omega\right)^{2}}\right)\right|_{\lambda_{i}}\right)\left.\right|_{\omega=-j / \lambda_{i}} \\
=\left.\left(\prod_{\substack{k=1 \\
k \neq i}}^{M} \frac{1}{\left(1-j \lambda_{k} \omega\right)^{2}}\right)\left(\sum_{\substack{m=1 \\
m \neq i}}^{M} \frac{2 j \lambda_{m}}{1-j \lambda_{m} \omega}\right)\left(\frac{j}{\lambda_{i}}\right)\right|_{\omega=-j / \lambda_{i}} .
\end{gathered}
$$

Consequently by letting $\omega=-j / \lambda_{i}$

$$
\begin{aligned}
B_{i} & =\left(\prod_{\substack{k=1 \\
k \neq i}}^{M} \frac{1}{\left(\lambda_{k} / \lambda_{i}-1\right)^{2}}\right)\left(\sum_{\substack{m=1 \\
m \neq i}}^{M} \frac{-2 \lambda_{m}}{\lambda_{i}-\lambda_{m}}\right) \\
& =A_{i} \sum_{\substack{m=1 \\
m \neq i}}^{M} \frac{-2 \lambda_{m}}{\lambda_{i}-\lambda_{m}} .
\end{aligned}
$$


Therefore

$$
\begin{aligned}
f_{\text {DPSA }}(t)= & \int_{-\infty}^{\infty} \prod_{i=1}^{M} \frac{1}{\left(1-j \lambda_{i} \omega\right)^{2}} \exp (-j \omega t) \frac{d \omega}{2 \pi} \\
= & \sum_{i=1}^{M} \int_{-\infty}^{\infty} \frac{A_{i}}{\left(1-j \lambda_{i} \omega\right)^{2}} \exp (-j \omega t) \frac{d \omega}{2 \pi} \\
& +\sum_{i=1}^{M} \int_{-\infty}^{\infty} \frac{B_{i}}{\left(1-j \lambda_{i} \omega\right)} \exp (-j \omega t) \frac{d \omega}{2 \pi} \\
= & \sum_{i=1}^{M} \frac{A_{i}}{2 \lambda_{i}^{2}} t \exp \left(\frac{-t}{\lambda_{i}}\right)+\sum_{i=1}^{M} \frac{B_{i}}{\lambda_{i}} \exp \left(\frac{-t}{\lambda_{i}}\right)
\end{aligned}
$$

$\mathscr{F}_{\text {DPSA }}(\gamma)$ results by integrating $f_{\text {DPSA }}(t)$ as

$$
\begin{aligned}
\mathscr{F}_{\text {DPSA }}(\gamma)= & \sum_{i=1}^{M}\left(A_{i}+B_{i}\right)-\sum_{i=1}^{M} B_{i} \exp \left(\frac{-\gamma}{\lambda_{i}}\right) \\
& -\sum_{i=1}^{M} A_{i} \exp \left(\frac{-\gamma}{\lambda_{i}}\right)\left(1+\frac{\gamma}{\lambda_{i}}\right) \\
= & 1-\sum_{i=1}^{M}\left(A_{i}\left(1+\frac{\gamma}{\lambda_{i}}\right)+B_{i}\right) \exp \left(\frac{-\gamma}{\lambda_{i}}\right) .
\end{aligned}
$$

\section{References}

[1] G. E. Corazza, C. Caini, A. Vanelli-Coralli, and A. Polydoros, "DS-CDMA code acquisition in the presence of correlated fading_part I: theoretical aspects," IEEE Transactions on Communications, vol. 52, no. 7, pp. 1160-1168, 2004.

[2] C. Caini, G. E. Corazza, and A. Vanelli-Coralli, "DS-CDMA code acquisition in the presence of correlated fading-part II: application to cellular networks," IEEE Transactions on Communications, vol. 52, no. 8, pp. 1397-1407, 2004.

[3] T. S. Rappaport, Wireless Communications: Principles and Practice, Prentice Hall, 2nd edition, 2002.

[4] J. Nielsen, S. K. Shanmugam, M. U. Mahfuz, and G. Lachapelle, "Enhanced detection of weak GNSS signals using spatial combining," Navigation, Journal of the Institute of Navigation, vol. 56, no. 2, pp. 83-95, 2009.

[5] A. Broumandan, J. Nielsen, and G. Lachapelle, "Indoor GNSS signal acquisition performance using a synthetic antenna array," IEEE Transactions on Aerospace and Electronic Systems, vol. 47, no. 2, pp. 1337-1350, 2011.

[6] J. K. Holmes and C. C. Chen, "Acquisition time performance of PN spread-spectrum systems," IEEE Transactions on Communications, vol. 25, no. 8, pp. 778-784, 1977.

[7] R. Watson, G. Lachapelle, and R. Klukas, "Testing oscillator stability as a limiting factor in extreme high-sensitivity GPS applications," in Proceedings of the European Navigation Conference, pp. 1-20, Manchester, UK, May 2006.

[8] B. Friedlander and S. Scherzer, "Beamforming versus transmit diversity in the downlink of a cellular communications system," IEEE Transactions on Vehicular Technology, vol. 53, no. 4, pp. 1023-1034, 2004.

[9] S. Hyeon, Y. Yun, H. Kim, and S. Choi, "Phase diversity for an antenna-array system with a short interelement separation,"
IEEE Transactions on Vehicular Technology, vol. 57, no. 1, pp. 206-214, 2008.

[10] D. G. Brennan, "Linear diversity combining techniques," in Proceedings of the Institute of Radio Engineers (IRE '59), vol. 47, pp. 1075-1102, June 1959.

[11] J. S. Colburn, Y. Rahmat-Samii, M. A. Jensen, and G. J. Pottie, "Evaluation of personal communications dual-antenna handset diversity performance," IEEE Transactions on Vehicular Technology, vol. 47, no. 3, pp. 737-746, 1998.

[12] W. C. Jakes, Microwave Mobile Communications, IEEE Press, Piscataway, NJ, USA, 1974.

[13] W. C. Y. Lee, Mobile Communication Engineering, McGraw-Hill, 2nd edition, 1997.

[14] A. M. D. Turkmani, A. A. Arowojolu, P. A. Jefford, and C. J. Kellett, "An experimental evaluation of the performance of twobranch space and polarization diversity schemes at $1800 \mathrm{MHz}$," IEEE Transactions on Vehicular Technology, vol. 44, no. 2, pp. 318-326, 1995.

[15] R. M. Narayanan, K. Atanassov, V. Stoiljkovic, and G. R. Kadambi, "Polarization diversity measurements and analysis for antenna configurations at $1800 \mathrm{MHz}$," IEEE Transactions on Antennas and Propagation, vol. 52, no. 7, pp. 1795-1810, 2004.

[16] C. B. Dietrich, K. Dietze, J. R. Nealy, and W. L. Stutzman, "Spatial, polarization, and pattern diversity for wireless handheld terminals," IEEE Transactions on Antennas and Propagation, vol. 49, no. 9, pp. 1271-1281, 2001.

[17] M. Zaheri, A. Broumandan, V. Dehgahanian, and G. Lachapelle, "Detection performance of polarization and spatial diversity for indoor GNSS applications," International Journal of Antennas and Propagation, vol. 2012, Article ID 879142, 10 pages, 2012.

[18] A. Broumandan, J. Nielsen, and G. Lachapelle, "Signal detection performance in rayleigh fading environments with a moving antenna," in Proceedings of the IEEE Transactions on Signal Processing, vol. 4, pp. 117-129, April 2010.

[19] S. Stergiopoulos and H. Urban, "A new passive synthetic aperture technique for towed arrays," IEEE Journal of Oceanic Engineering, vol. 17, no. 1, pp. 16-25, 1992.

[20] V. Dehghanian, J. Nielsen, and G. Lachapelle, "Combined spatial-polarization correlation function for indoor multipath environments," IEEE Antennas and Wireless Propagation Letters, vol. 9, pp. 950-953, 2010.

[21] V. Dehghanian, J. Nielsen, and G. Lachapelle, "Merger of polarization and spatial diversity by moving a pair of orthogonally polarized dipoles," in Proceedings of the 23rd Canadian Conference on Electrical and Computer Engineering (CCECE '10), Calgary, Canada, May 2010.

[22] V. Dehghanian, M. Zaheri, J. Nielsen, and G. Lachapelle, "Dual-polarized synthetic array for indoor GNSS handheld applications," in Proceedings of the 7th International Symposium on Wireless Communication Systems (ISWCS'10), pp. 661-665, September 2010.

[23] H. L. VanTrees, Detection Estimation and Modulation Theory: Part IV, John Wiley \& Sons, New York, NY, USA, 2002.

[24] D. A. Hill and J. M. Ladbury, "Spatial-correlation functions of fields and energy density in a reverberation chamber," IEEE Transactions on Electromagnetic Compatibility, vol. 44, no. 1, pp. 95-101, 2002.

[25] B. Friedlander and S. Scherzer, "Beamforming versus transmit diversity in the downlink of a cellular communications system," IEEE Transactions on Vehicular Technology, vol. 53, no. 4, pp. 1023-1034, 2004. 
[26] S. Kay, Fundamentals of Statistical Signal Processing: Detection Theory, Prentice Hall, 1993.

[27] W. C. Y. Lee, "Estimate of local average power of a mobile radio signal," IEEE Transactions on Vehicular Technology, vol. 34, no. 1, pp. 22-27, 1985.

[28] S. Y. Seidel and T. S. Rappaport, "914 MHz path loss prediction models for indoor wireless communications in multifloored buildings," IEEE Transactions on Antennas and Propagation, vol. 40, no. 2, pp. 207-217, 1992. 

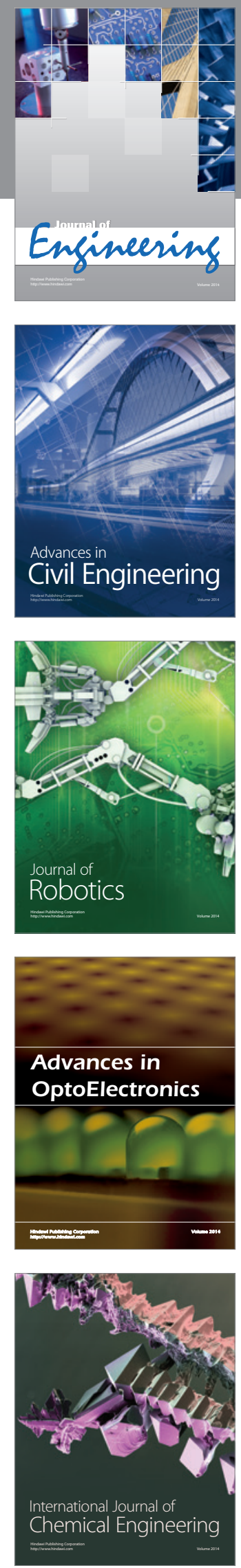

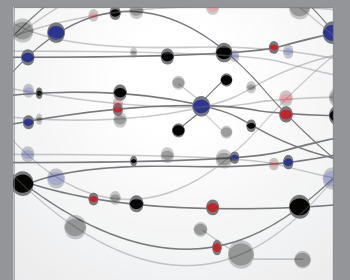

The Scientific World Journal
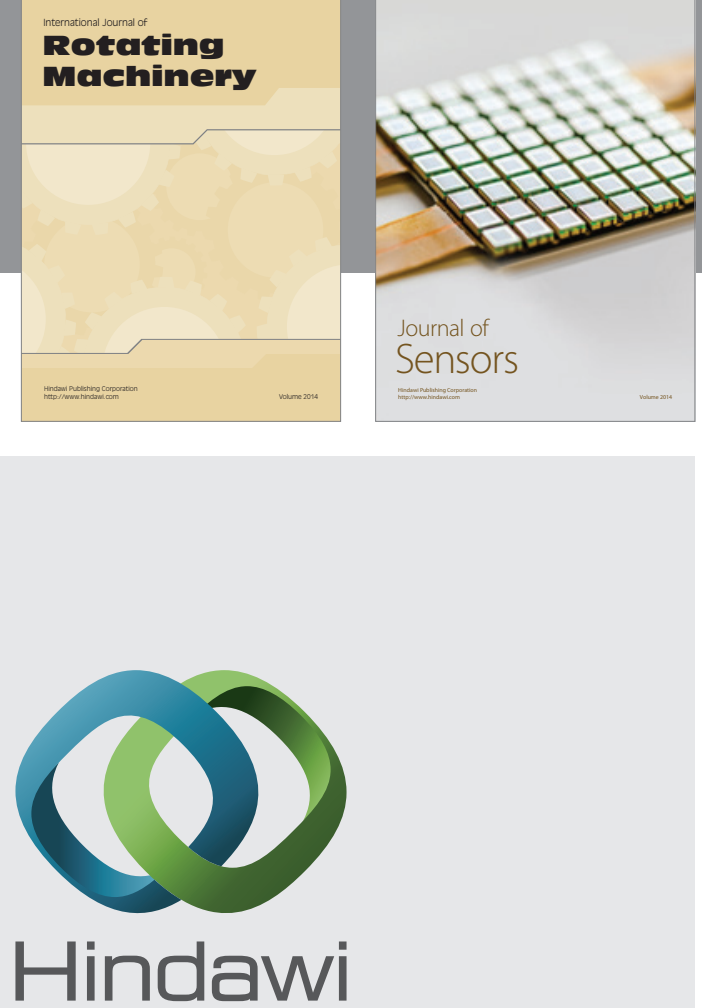

Submit your manuscripts at http://www.hindawi.com
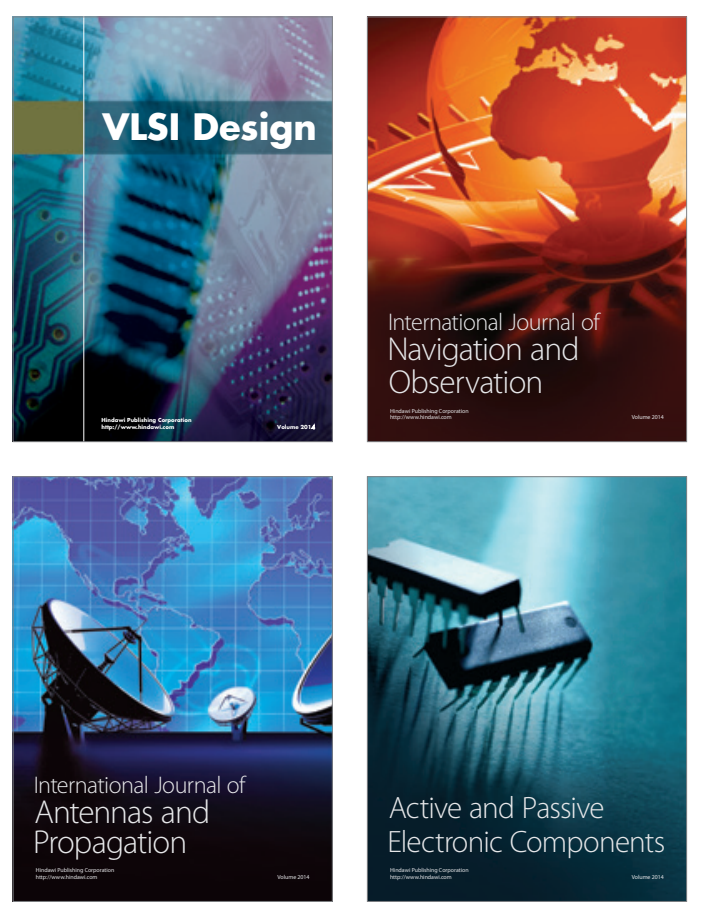
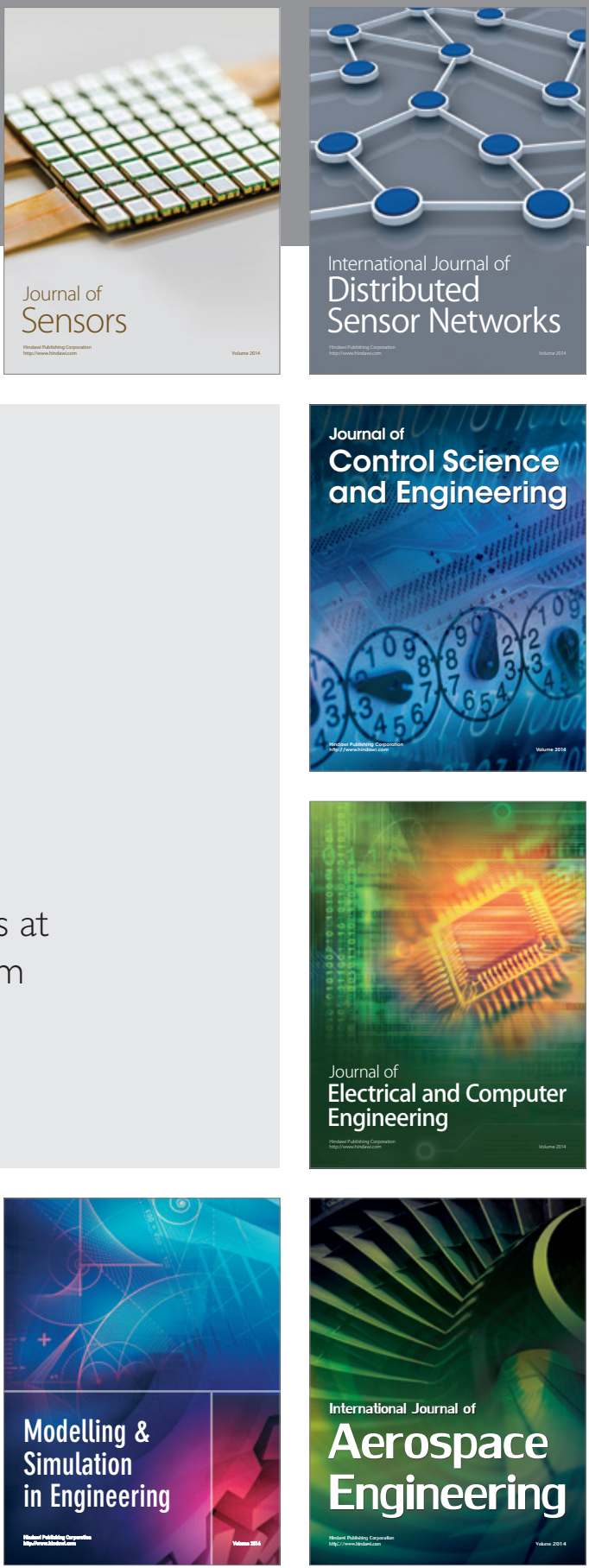

Journal of

Control Science

and Engineering
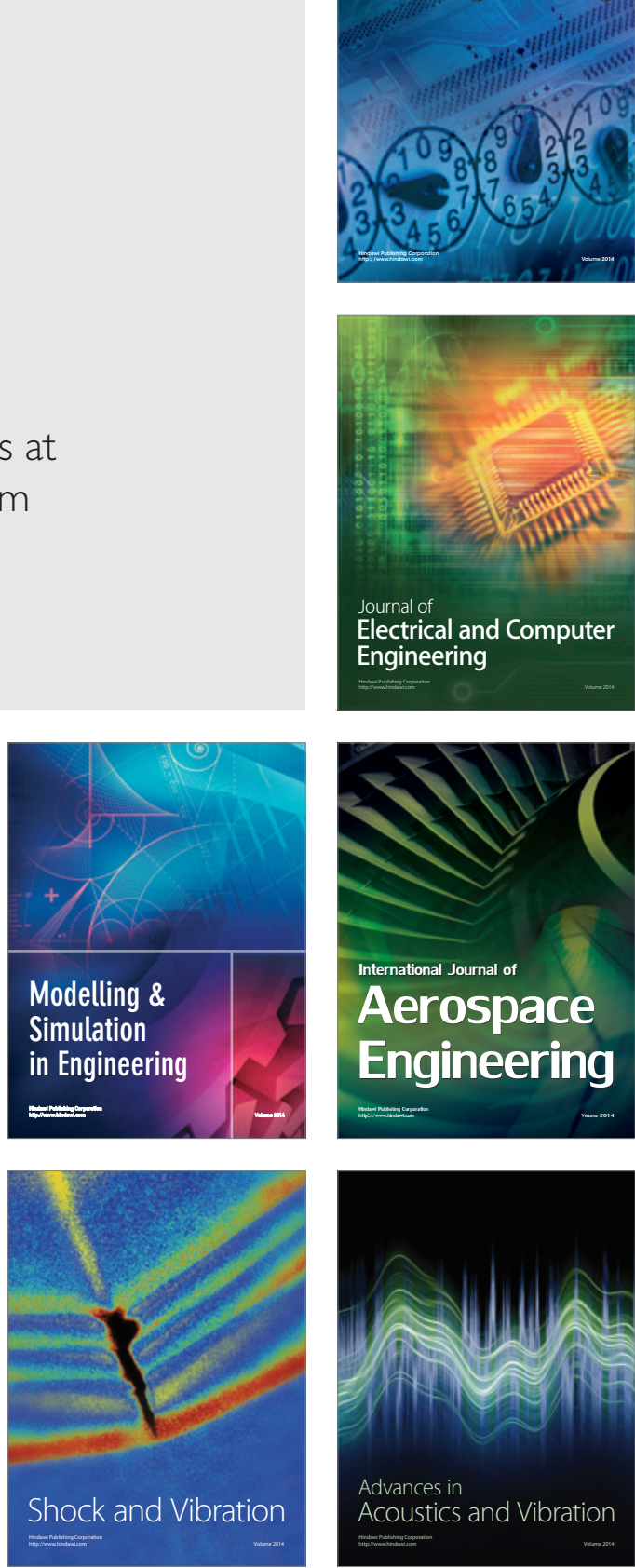